\title{
Sphingolipidome quantification by liquid chromatography- high resolution mass spectrometry: whole blood vs. plasma
}

\author{
Dezhen Wang ${ }^{1}$, Peining $\mathrm{Xu}{ }^{1}$ and Clementina Mesaros ${ }^{1, *}$ \\ 1 Center for Excellence in Environmental Toxicology, Department of Systems Pharmacology and Translational \\ Therapeutics, University of Pennsylvania, Philadelphia, PA 19104 \\ * Correspondence: Clementina Mesaros; E-mail: mesaros@upenn.edu; Phone: 215-573-9886; Fax: 215-573-9889
}

Citation: Wang, D..; Xu, P..;

Mesaros, M. Sphingolipidome quantification by liquid chromatography- high resolution mass spectrometry: whole blood vs. plasma. Metabolites 2021, 11, x. https://doi.org/10.3390/xxxxx

\begin{abstract}
Plasma and serum are the most widely used blood-derived biofluids for metabolomics and lipidomics assays, but the isolation of these products from blood may introduce additional bias as indicated by the fact that many analytes that are present at high concentrations in blood cells cannot be measured and evaluated in those samples. Of particular concern, variable hemolysis during the pre-processing of blood products could compromise accurate and reproducible quantification. Compared with plasma or serum, whole blood may be a better alternative due to simplicity of processing. In this study, we provide a comprehensive method for quantification of the whole blood sphingolipidome and the concentrations were compared with those from plasma. Combining a single-phase extraction method with liquid-chromatography high resolution mass spectrometry ( $R=120,000)$, assisted by alkaline hydrolysis, we were able to identify and simultaneously quantify more than 150 sphingolipids. Furthermore, most of sphingolipids remained stable after a freeze/thaw cycle. Whole blood contained a higher concentration of most sphingolipids than corresponding plasma. Moreover, individual variations in the levels of sphingolipids were lower for whole blood than plasma. These findings demonstrate that whole blood could be a better alternative to plasma, and potentially guide the evaluation of sphinglipidome for biomarker discovery.
\end{abstract}

Keywords: sphingolipidome; ceramides; high resolution mass spectrometry; whole blood; plasma

\section{Introduction}

Blood consists of different components, including red blood cells, white blood cells, platelets, and plasma. In conventional metabolomics studies, plasma or serum derived from whole blood is used for the analysis, which could underestimate a lot of metabolic pathways, such as glycolysis, coenzymes, antioxidants, and purine metabolism. Those metabolic intermediates are usually present at high concentrations in blood cells but are not detected (or are very low) in plasma[1,2]. Increasing evidence demonstrated that whole blood metabolomics could provide more useful metabolites information in delineating metabolic effects related to aging, disease, nutrition, and environmental stressors than plasma metabolomics[3-5]. One notable example of the use of whole blood is the quantification of Acetyl-CoA, as its detection was impossible in serum or plasma[6] together with a high-throughput method for quantitative detection of eicosanoids in human whole blood and fatty acids[3,7]. Plasma "omics" measurements have a large degree of variability from plasma isolation, so freezing the whole blood as soon as was drawn could improve this variability.

To our knowledge, limited studies used whole blood for lipidomics analysis using liquid-chromatography high resolution mass spectrometry (LC-HRMS)[8-12] but there are several reports using ${ }^{1} \mathrm{H}-\mathrm{NMR}$, which has by far less sensitivity when compared with LC-MS. In order to assess the advantage of using whole blood for lipidomics analysis, the lipid composition should be elucidated and compared with plasma. Marasca et al.[8] have chosen 15 lipids species from most of lipids classes and compared their profiles 
from whole blood, dry blood spots (DBS) or volumetric absorptive microsampling (VAMS).

The whole blood lipidomics analysis yields a very rich data set so we focused our initial study on sphingolipids. They are one of the major classes of eukaryotic lipids that are forming an outer leaflet of the plasma membrane lipid bilayer, and a lot of bioactive sphingolipids, such as ceramides (Cer), sphingosine-1-P, and glycosphingolipids, are supposed to be involved in many aspects of cellular functions, including cell proliferation, cell differentiation, apoptosis, cell cycle arrest, senescence, autophagy, cytoskeleton rearrangement, necrosis, inflammation, neurodegeneration, and cancer cell migration and invasion[13-16].Their synthesis and degradation processes[17-19] have different intracellular compartmentalization, including the endoplasmic reticulum (ER), Golgi apparatus and lysosomes.

To potentially guide the evaluation of sphinglipidome in blood for biomarker discovery, we developed a comprehensive method for quantification of sphingolipids in whole blood using single phase extraction method combined with high resolution mass spectrometry. We provided robust annotations and quantification assisted by alkaline hydrolysis, parallel reaction monitoring (PRM)/ data-independent acquisition (DIA), and appropriate internal standard normalization. One freeze/thaw cycle had no significant effect of sphingolipids, which will improve the option to use stored whole blood for sphingolipid analysis. More importantly, we compared the sphingolipidome of whole blood and paired plasma, showing that whole blood contained a higher concertation of most sphingolipids than the paired plasma, making the analysis accessible to less sensitive methods. Reducing the processing steps of the whole blood during plasma or serum isolation by directly freezing the whole blood at $-80{ }^{\circ} \mathrm{C}$ may provide a more robust workflow for large lipidomics studies and allow for a more complete picture of the whole metabolism.

\section{Results and Discussions}

2.1 Single phase extraction combined with high resolution mass spectrometry provides accurate quantification of whole blood sphingolipidome

Due to the lack of tested methods for whole blood lipidomics, we selected several extraction protocols that were reported for plasma or serum (MTBE two phase, MTBE single phase, butanol single phase) and compared the fortified extraction efficacies of different sphingolipid internal standards. All three methods showed good recoveries for ceramides, ceramide-1-P, sphingomyelins, and hex-ceramides (Figure 1A). However, the two-phase MTBE methods showed poor recoveries for sphingosine, sphinganine and their phosphate forms. The modified MTBE method enhanced the recovery of sphingosine-1-P and sphinganine-1-P but showed little improvement for sphingosine and sphinganine. Compared with the MTBE method, the single-phase (SP) butanol method, which was recently developed for plasma and serum lipidomics, exhibited good recoveries for all the sphingolipids (Figure 1A, red bars). Therefore, the single-phase butanol extraction method was used for sample preparation of the whole blood sphingolipidome.

To accurately quantify the sphingolipids, we set the resolution of MS1 as high as $120,000 @ m / z 200$. This extreme high resolution can achieve a good accuracy with a mass error of less than 1ppm for most of sphingolipids (Figure 1B). Even for the GM3(d34:1), with $m / z$ higher than 1000, the resolution at $m / z 1153.7217$ was 53,202 with mass accuracy around 1ppm (Figure S1). We also acquired MS2 fragmentations using data dependent acquisition (DDA) in both positive and negative mode (separately). The positive mode provided the polar lipid groups, such as SPH(d18:0) (Figure 1C), while the negative mode provided additional information about fatty acid chain length (Figure 1D). Combining the MS1 precursor with the MS2 fragments identification, we were able to positively identify all sphingolipid classes and typical spectra for each class in both ionization modes are included in the Supporting Information Figures S2-S6. 

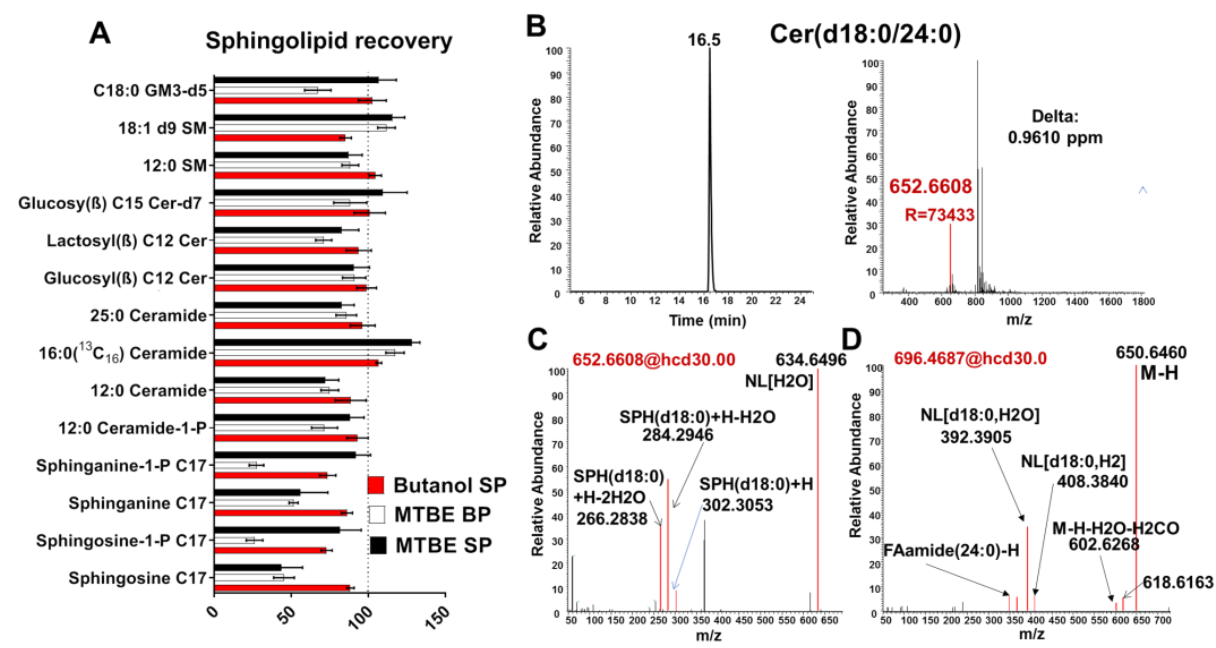

Figure 1. Single phase extraction combined with high resolution mass spectrometry provides accurate quantification of whole blood sphingolipidome. (A) Comparison of fortified recoveries of sphingolipids from three different extraction methods (Butanol single phase-SP, MTBE bi-phase-BP, and MTBE single phase-SP). (B) The MS1 high resolution $(R=120,000)$ used for quantification of sphingolipids showed a high accuracy for Cer(d18:0/24:0). Both (C) positive $[\mathrm{M}+\mathrm{H}]^{+}$and $(\mathrm{D})$ negative $[\mathrm{M}-\mathrm{HCOO}]^{-}$mode are used for the structure elucidation of sphingolipid Cer(d18:0/24:0).

\subsection{Alkaline hydrolysis improves the annotation of sphingolipids}

Using DDA method, only the top 20 mass were selected for collision induced dissociation to acquire the MS2 fragment. For the low abundance metabolites or the ones masked by high abundant phospholipids was difficult to acquire their MS2 information. Alkaline hydrolysis was previously used to eliminate abundant phospholipids and to facilitate the annotation and quantification of sphingolipids[20,21]. However, comprehensive evaluation of stability of sphingolipids under alkaline condition was rarely performed. We fortified the extraction solvent with different sphingolipid internal standards and compared the peak intensity before and after alkaline hydrolysis $\left(0.1 \mathrm{M} \mathrm{KOH}\right.$ at $37^{\circ} \mathrm{C}$ for $45 \mathrm{~min}$ ). Consistent with previous results, most of sphingolipids, including ceramides, ceramide-1-P, hex-ceramides, and sphingomyelins remained stable after alkaline hydrolysis[22] (Figure 2A). Sphinganine and sphingosine levels significantly decreased after alkaline treatment, which was also found in other solvent systems, such as $\mathrm{CH}_{2} \mathrm{Cl}_{2} /$ methanol (data not shown) and are worth investigating more. However, due to the presence of their corresponding internal standards, this should not be a major problem.

Alkaline hydrolysis facilitates the annotation of sphingolipids in two different ways, similar to the case of plasma analysis[22]. Firstly, it improves the annotation of sphingolipids by allowing for acquiring more MS2 fragmentation. For examples, Cer(m18:0/24:0) (Figure 2B) showed similar intensity before (black peak) and after alkaline treatment (red peak), but only the alkaline treated samples showed the corresponding MS2 spectrum, which was annotated by LipidSearch with their Product search strategy. Secondly, the alkaline treatment hydrolyzed most of the phosphocholines (one of the best ionizing lipids that elute at similar retention times with most sphingolipids), so more sphingomyelins could be annotated based on their specific polar head fragment $\mathrm{m} / \mathrm{z}$ 184.0732. Even combining this strategy with the HRMS, some of the fatty acid chains could not be completely annotated.

Combining the very high resolution for MS1 and the MS2 fragmentation, assisted by alkaline hydrolysis, more than 140 sphingolipids were identified and annotated, including 46 ceramides (Figure 2C). The 46 ceramides belonged to three major classes: ceramides (30), deoxyceramides (9), and phytoceramides (5) (Figure 2D). 


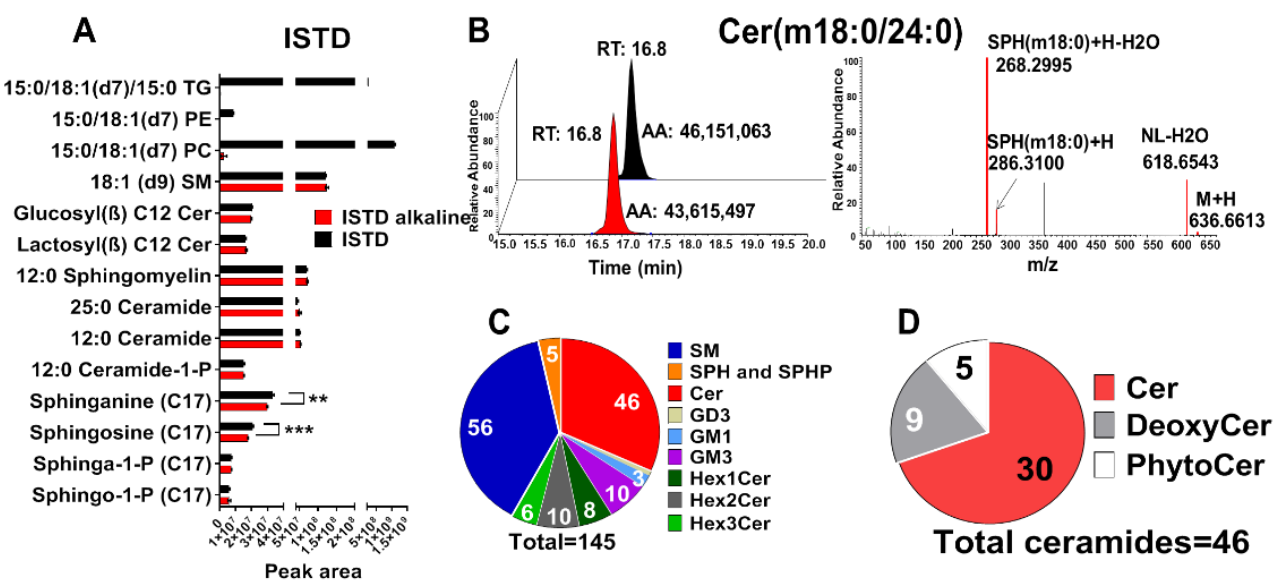

Figure 2. Alkaline hydrolysis facilitates the annotation of sphingolipids in whole blood. (A) The effects of alkaline hydrolysis $(0.1 \mathrm{M} \mathrm{KOH}$ for $45 \mathrm{~min})$ on intensities of sphingolipids, phospholipids, and triglycerides. (B) The alkaline hydrolysis had no enhancement of intensity of Cer(m18:0/24:0) (red peak, after alkaline hydrolysis) but in Date Dependent Acquisition (DDA) mode does improve the annotation of the spectrum of Cer(m18:0/24:0). (C) The 145 total annotated sphingolipids including Cer, ganglioside (GD3, GM1, and GM3), HexCer (Hex1Cer, Hex2Cer, Hex3Cer), SM, SPH, and SPHP. (D) The annotated ceramides can be categorized into three main subclasses Cer, DeoxyCer, and PhytoCer.

\subsection{Inclusion list based PRM/DIA improves the annotation of isobaric subspecies of ceramides}

Butanol single phase extraction combined with ddMS2 (DDA) annotated more than 140 sphingolipids, which was used to generate the inclusion list with scheduled scan windows (1 min), and the inclusion list was then used for PRM/DIA to resolve the isobaric subspecies of several ceramides (Figure 3A). For examples, Cer(d44:1) was present as different subspecies in whole blood, and the high abundant form Cer(d18:1/26:0) could be detected and easily annotated in DDA mode, while the small peak from Cer(d19:1/25:0) was not completely elucidated. PRM/DIA with inclusion list could selectively fragment Cer(d19:1/25:0) to acquire its representative fragmentation $\mathrm{SPH}(\mathrm{d} 19: 1)$, in either PRM (Figure 3B and 3C) or DIA mode (Figure S7). Moreover, some co-eluting ceramide were partially separated with the aid of DIA or PRM. For examples, Cer(d18:1/24:2) and Cer(d18:2/24:1) eluted at very similar retention time, but quantification based on their representative fragment could partially distinguish the two subspecies (Figure 3D). Using inclusion list based PRM and DIA, 8 more ceramides and one more Hex1Cer were identified together with 4 ceramide-1-P (Figure S8). Ceramide-1-P are important signaling molecules so their identification could be particularly useful. The final method identified 159 sphingolipids (Figure 3E), including 54 ceramides with different long-chain bases (LCB) (d18:0, d18:1. d18:2, d19:1, d20:1, m18:0, m18:1, m19:0, and t18:0) (Figure 3F). In the last decade the "a-typical" ceramides- not biosynthesized starting from Palmitoyl-CoA- started to be reported as relevant in diverse model organisms. To cross check the annotated results, authentic standards were used for some sphingolipids to verify retention time and the MS2 spectra. These standards included Cer(d18:1/16:0), Cer(d18:0/16:0), Cer(d18:1/18:0), Cer(d18:0/18:0), Cer(d18:1/24:0), Cer(d18:0/24:0), Cer(d18:1/24:1), Cer(18:0/24:1), Cer(18:2/24:0), sphinganine, sphingosine, sphiganine-1-P, sphingosine-1-P, CerP(d18:1/24:0), SM(d18:1/16:0), and SM(d18:1/24:0). 


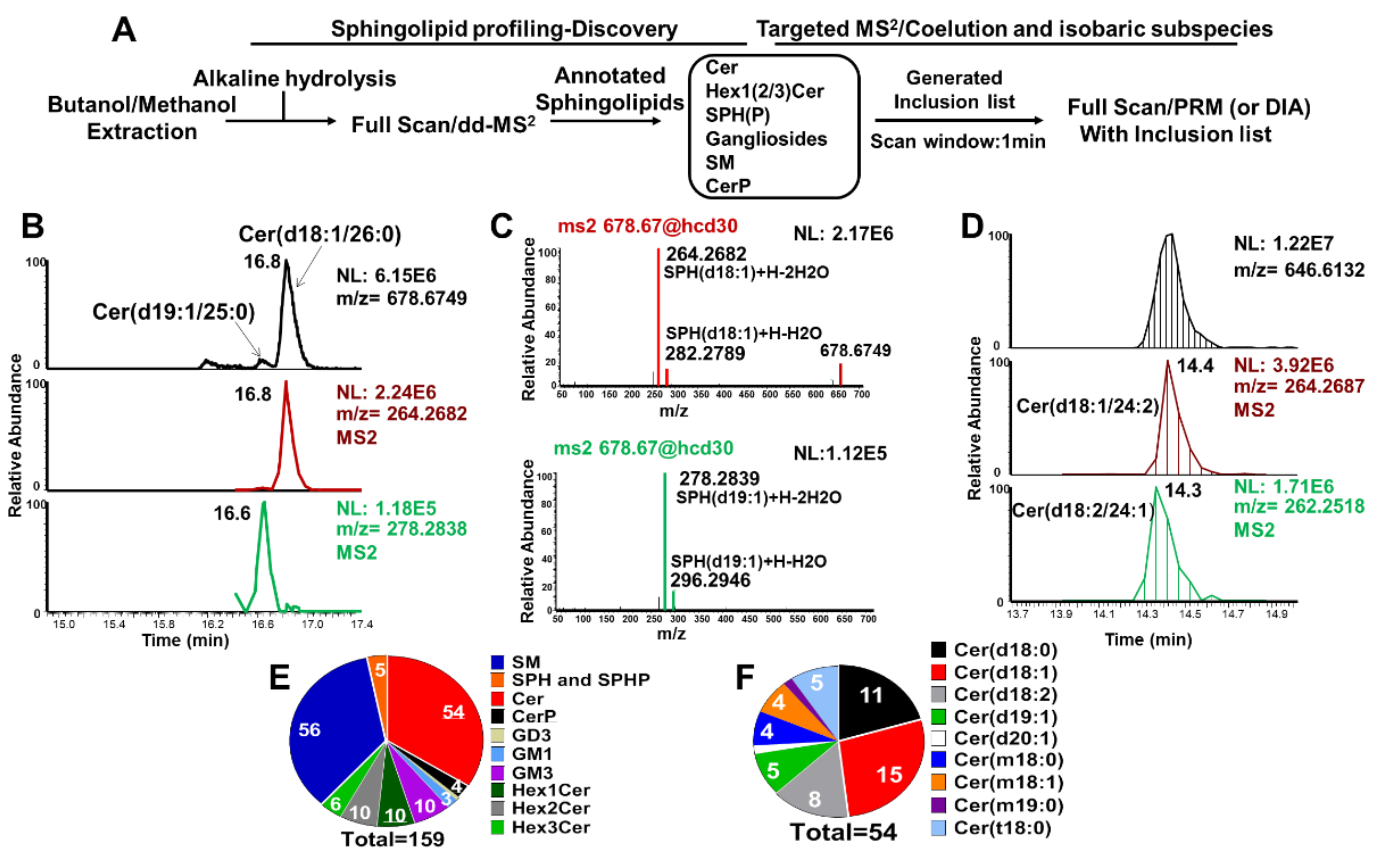

Figure 3. Inclusion list based PRM/DIA improves the annotation of isobaric subspecies of ceramides. (A) The schematic graph shows the strategy used for PRM/DIA analysis. (B) The isomers of Cer(d44:1) were annotated by PRM through acquiring the MS2 spectrum of the low abundant Cer(19:1//25:0). (C) MS2 spectra of Cer(d18:1/26:0) and Cer(19:1/25:0) showed their representative distinctive fragments. (D) Co-eluting isomers Cer(d18:1/24:2) and Cer(d18:2/24:1) were separated by their representative fragments in DIA mode, (E) The final annotated sphingolipids improved by PRM/DIA to 159,(F) 54 total ceramides were quantified with different acyl chains.

Compared with previous studies, the current methodology identified and quantified the most of ceramides (54 total), a number similar or higher when compared with targeted MRM based methods[23,24]. More importantly, the high resolution-DDA/DIA/PRM methods have a high accuracy, which will be superior to the targeted lipidomics and allows for further data investigation.

\subsection{Appropriate internal standards corrections eliminate matrix effects and achieves high dynamic linearities}

Matrix effects[25] have been observed in LC-MS methods with a dramatic loss in response (ion suppression) for higher volumes of whole blood. Figure 4A shows the Cer(d18:1/24:1) peak intensities from different volumes of whole blood. When the volume of blood is higher than $50 \mu \mathrm{L}$, the peak response versus volume is not linear anymore. To eliminate the matrix effects that would interfere with quantification, we tested the ability of the internal standards from the Cer/Sph Mixture I (commercially available from Avanti ${ }^{\circledast}$ Polar Lipids), as the normalization factor for each class. For ceramides, both Cer (d18:1/12:0) and Cer (d18:1/25:0) were compared. Cer (d18:1/12:0) normalization corrected the response due to the ion suppression for most of (dihydro)ceramides, while 25:0 ceramide had no significant improvement (Figure $4 B)\left(R^{2} 0.99\right.$ vs $\left.R^{2} 0.88\right)$. After normalization with Cer (d18:1/12:0), most of the ceramides achieved good linearities with $\mathrm{R}^{2}>0.98$ (Figure S9 and S10). Due to the low natural abundance of Cer (d18:1/12:0), the Cer/Sph Mixture I could be supplemented with a labeled ceramide like [ $\left.{ }^{13} \mathrm{C} 16\right]$-Cer (d18:1/16:0) (available from Cambridge Isotopes Labs), but the concentration of Cer(d18:1/12:0) in the internal standard mixture was couple of orders of magnitude higher than the endogenous one, hence did not influence the normalization. The 17-base sphingosine phosphate (SPHP) showed a good normalization for SPHP (Figure 
S11). Normalization of the hexosyl-ceramides with Hex1Cer(d18:1/12:0) could not minimize the matrix effect for Hex1Cer (Figure 4C, middle panel). Normalization of the SM (d34:1) peak area with the SM (d18:1/12:0) from the Cer/Sph mix (Figure 4D, middle panel) looked even worse compared with the peak area without normalization. To correct for these limitations, we added other stable isotope internal standards to correct for some classes: gangliosides (added C18:0 GM3-d5), Hex-Cer (added Hex1Cer(d18:1-d7/15:0)) and SM (added SM(d18:1/18:1-d7)). Hex1Cer(d18:1-d7/15:0) normalization improved the linearity of most of hex-ceramides with $\mathrm{R}^{2}>0.98$ (Figure $4 \mathrm{C}$ and Figure S12, S13, and S14). SM(d18:1/18:1-d7) and C18:0 GM3-d5 were able to minimize the matrix effects for most of SM (Figure 4D and Figure S15) and gangliosides (Figure S16). In conclusion, by selecting the appropriate internal standards for normalization for each class, including added labeled standards like SM(d18:1/18:1-d7), Hex1Cer(d18:1-d7/15), 18:0 GM3-d5 the matrix effects were minimized, and high dynamic linearity was achieved.
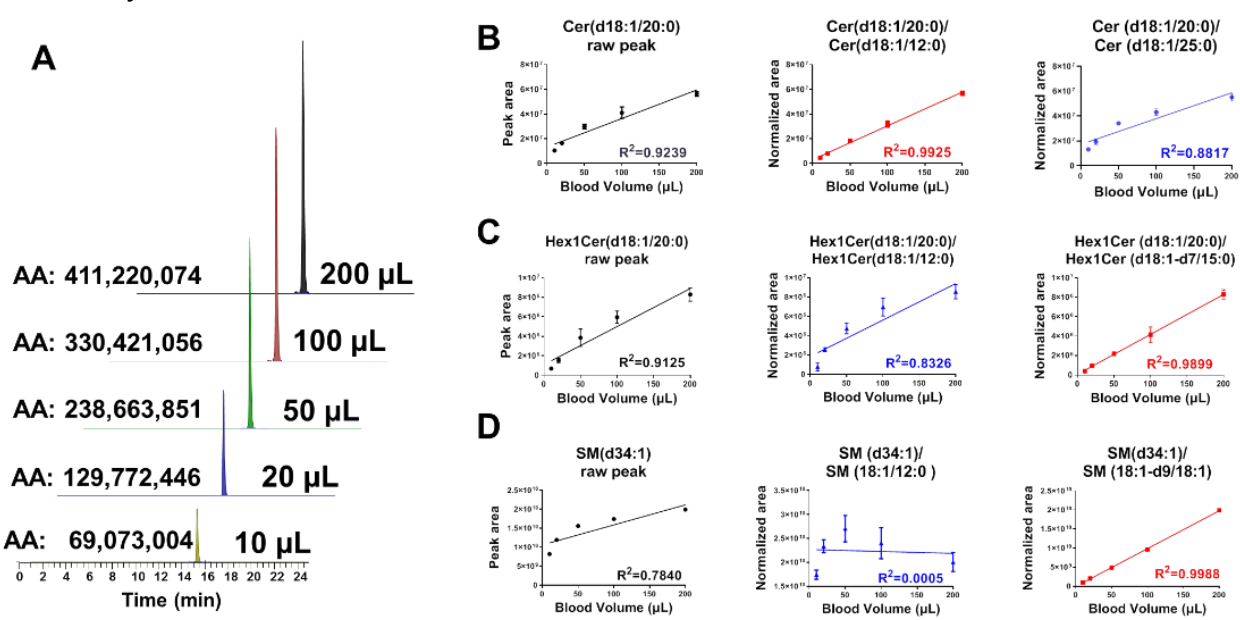

D
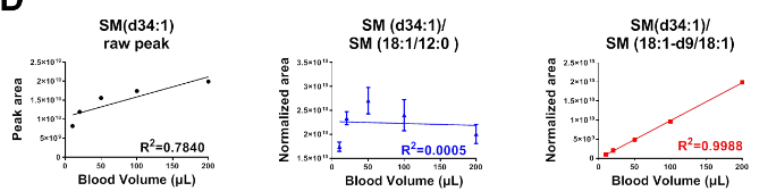

Figure 4. Appropriate internal standard correction achieves a high dynamic linearity between peak intensity and whole blood volume. (A) No correlation between peak intensity and the blood volume, implys big matrix effects for higher volumes of blood. (B) Comparison of the raw peak intensity or normalized intensity (internal standard 12:0 ceramide or 25:0 ceramide) of Cer(d18:1/20:0), and their linearities against blood volume. (C) Comparison of the raw peak intensity or normalized intensity (internal standard 12:0 Hex1Cer or C15 Hex1Cer-D7) of Hex1Cer(d18:1/20:0), and their linearities against blood volume. (D) Comparison of the raw peak intensity or normalized intensity (internal standard 12:0 SM or 18:1 d9 SM) of SM(d34:1), and their linearities against blood volume.

\subsection{Freeze/thaw cycle will not influence the stability of sphingolipids}

One disadvantage that could restrict the potential widespread application of whole blood in clinical metabolomics or lipidomics is the stability of metabolites in whole blood, which may be impacted by the enzymatic activity induced by the cell lysis during freeze/thaw cycle. Previous studies have reported some metabolites such acetyl-CoA may encounter dramatic loss during the thawing process[6], however, no data about the stability of sphingolipids in whole blood have been reported so far. Most of sphingolipids have been reported stable in plasma or serum at low temperatures[26]. One study reported that ceramides could be stable at room temperature for 8h[27]. To test the freeze/thaw stability of sphingolipids in whole blood samples, sphingolipids extracted from fresh collected whole blood (within $1 \mathrm{~h}$ after blood draw) were compared with the sphingolipid levels after freeze (at $-80^{\circ} \mathrm{C}$ ) or freeze and thaw (Figure 5A). Surprisingly, the major sphingolipids, including SPHP, ceramides, hex-ceramides, and sphingomyelins showed no significant decrease after freeze or the freeze/thaw cycle (Figure 5B). These results demonstrated that whole blood could be directly used for the sphingolipidome analysis. 
A

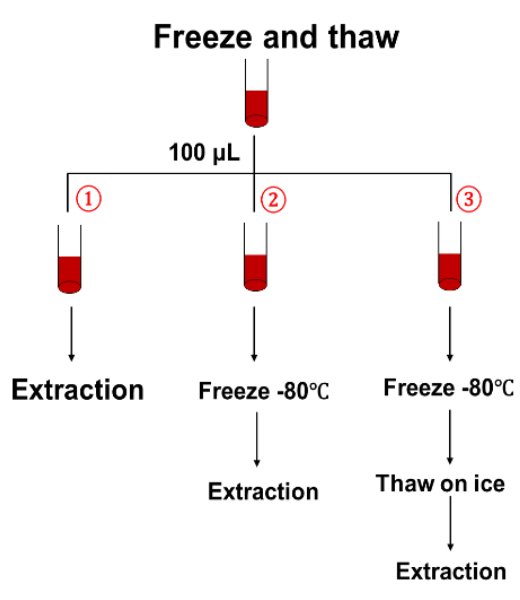

B

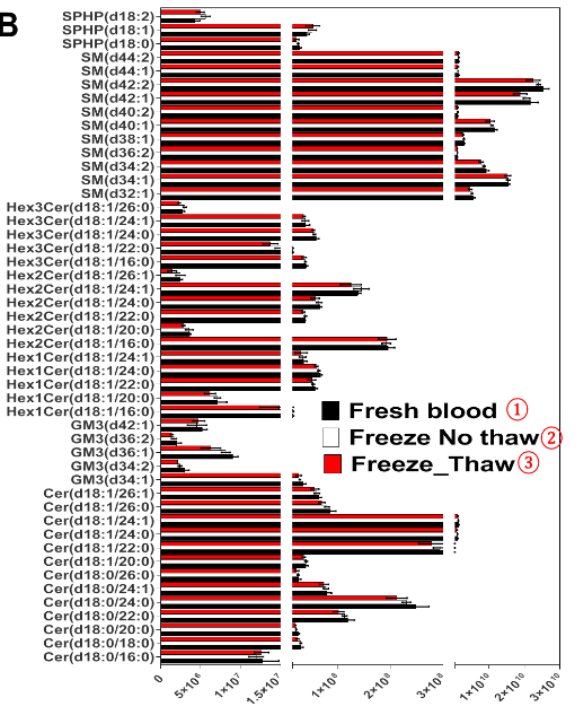

Figure 5. Freeze/thaw cycle does not influence the intensity of sphingolipids in whole blood. (A) Schematic diagram of freeze/thaw experiment for sphingolipid stability in whole blood. (B) Comparison of the peak intensities of major sphingolipids in fresh blood and freeze/thawed blood.

\subsection{Whole blood has a richer abundance of sphingolipids compared with paired plasma}

The sphingolipidome of 10 whole blood samples and 10 paired plasma samples from healthy donors was performed based on the butanol single-phase extraction coupled with the LC-HRMS method developed for the analysis of the whole blood sphingolipids. Compared with plasma, whole blood contained higher levels of ceramides (Figure 6A). The total concentration of ceramides in whole blood is nearly two-fold of that in plasma (Figure 6B). The gangliosides GD3 and GM1 were higher in whole blood, while gangliosides GM3 were higher in plasma (Figure 6A), which led to no significant difference of total gangliosides between whole blood and paired plasma (Figure 6B). For glycosylceramides, Hex1Cer (glucosylceramide and galactosylceramide) were lower in whole blood than paired plasma, while Hex2Cer and Hex3Cer were dramatically higher in whole blood, especially for Hex2(3)Cer(d18:1/26:0) and Hex2(3)Cer(d18:1/26:0) that were barely detected in plasma samples (Figure 6A and 6B). Sphingomyelins were the most abundant sphingolipids either in whole blood or plasma, but the whole blood has a much higher level of sphingomyelins (Figure 6A and 6B). SPH(P) especially sphingosine-1-P are important small bioactive molecules, and both SPH an SPHP were 2-3 times higher in whole blood than paired plasma (Figure 6A and 6B). Ceramide-1-P, another class of bioactive sphingolipids, also showed higher levels in whole blood, and not all plasma samples had detectable levels of ceramide-1-P using our LC-HRMS method. In conclusion, except for Hex1Cer and gangliosides, whole blood has a higher concentration of sphingolipids, including ceramide, CerP, Hex2Cer, Hex3Cer, SM, SPH, and SPHP. The lower abundance of the two classes would still allow for easy quantification (low $\mu \mathrm{M}$ levels) but for the sub- $\mu \mathrm{M}$ sphingolipids, any increase in abundance would help in the ease of quantification. After determining the corresponding internal standards, whole blood volume and freeze/thaw stability, we will investigate in further studies the contribution of each component of whole blood (red blood cells and white blood cells) to total blood sphingolipidome. Platelets sphingolipids will be determined by the difference. 
A

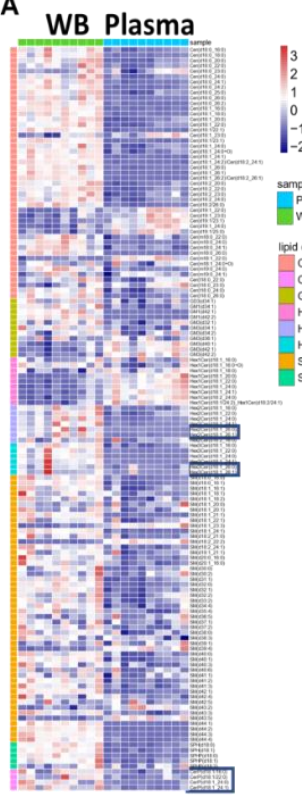

B

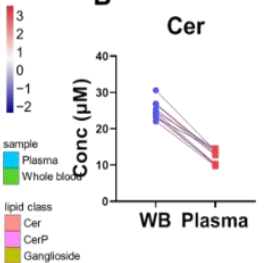

Hex2Cer
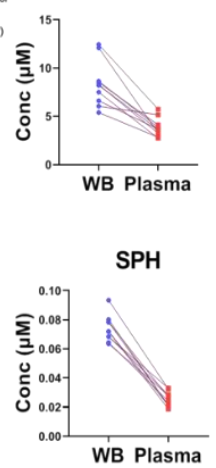

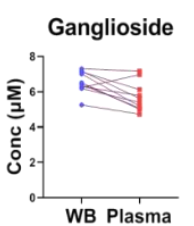

WB Plasma

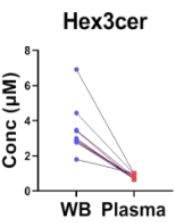

SPHP

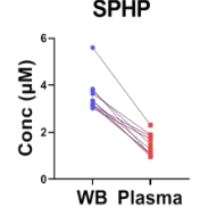

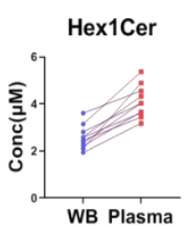

C

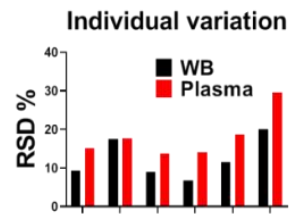

SM

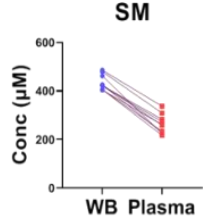

D
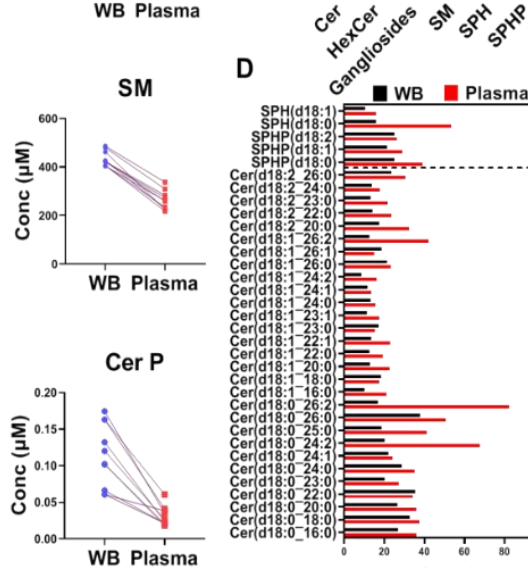

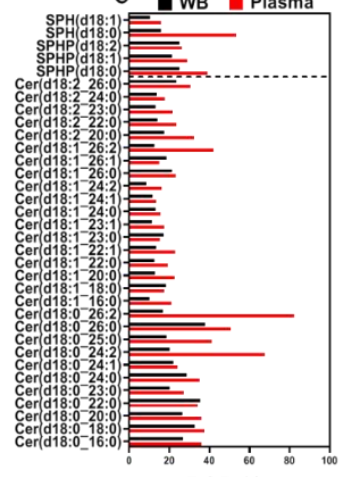

Figure 6. Whole blood (WB) and paired plasma sphingolipidome. The heatmap showed the comparison of major sphingolipids, including Cer, Ganglioside, Hex1Cer, Hex2Cer, Hex3Cer, SM and SPH(P) between WB and paired plasma from healthy donators (A). The total intensity of respective sphingolipid class in WB and paired plasma (B).

Apart from containing higher abundant sphingolipids, whole blood also showed advantages in the individual variations of sphingolipid levels in 10 paired samples. For most of sphingolipids, except for HexCer, lower values (RSD) of individual variations were observed for whole blood in comparison with plasma (Figure 6C). Specifically, some lower levels of sphingolipids, such as sphinganine, Cer(d18:0/24:2), Cer(d18:0/26:2), Cer(d18:1/26:2) showed much lower RSDs in whole blood compared with plasma, which may be due to the increased intensity and the ease of pre-process of whole blood.

One aspect that needs to be considered when using the volume of whole blood for analysis is the hematocrit- it measures the volume of red blood cells (RBC) compared to the total blood volume (red blood cells, white blood cells, platelets and plasma). The normal hematocrit for men is $40-54 \%$ and for women it is 36 to $48 \%$. Correction for the hematocrit bias in dried blood spot was detailed before [28] and the hematocrit levels can be easily obtain from the hemoglobin levels using formula HCT $=2.941$ hemoglobin conc $\mathrm{g} / \mathrm{dL}$ [29]. Hemoglobin can be easily measured using the Darbkin reagent and UV detection, using only $10 \mu \mathrm{L}$ of frozen whole blood[30]. Using the hemoglobin measurements would be easy to calculate the sphingolipids levels in erythrocytes as was shown before for folates [31].

\section{Materials and Methods}

\subsection{Chemicals and reagents.}

Acetonitrile $\left(\right.$ Optima $\left.^{\mathrm{TM}}\right)$, methanol $\left(\right.$ Optima $\left.{ }^{\mathrm{TM}}\right)$, water $\left(\right.$ Optima $\left.^{\mathrm{TM}}\right)$, MTBE (Opti$\mathrm{ma}^{\mathrm{TM}}$ ), 2-propanol (Optima $\left.{ }^{\mathrm{TM}}\right)$, methyl tert-butyl ether (MTBE) (HPLC grade), 1-butanol and formic acid (Optima ${ }^{\mathrm{TM}}$ ) were obtained from Fisher scientific (Waltham, MA, USA). Ammonium formate solution and potassium hydroxide were from Sigma-Aldrich (St. Louis, MO, USA). C18:0 GM3-d5, C15 Glucosyl( $\beta$ ) Ceramide-d7 (d18:1-d7/15:0), Cer/Sph Mixture I (Catalog No. LM6002) containing sphingosine (C17 base), sphinganine (C17 base), sphingosine-1-P (C17 base), sphinganine-1-P (C17 base), lactosyl(ß) C12 ceramide (Hex2Cer d18:1/12:0), 12:0 sphingomyelin (12:0 SM), glucosyl(ß) C12 ceramide (Hex1Cer d18:1/12:0), 12:0 ceramide (d18:1/12:0), 12:0 ceramide-1-P (CerP d18:1/12:0), and 25:0 Ceramide (d18:1/25:0), and SPLASH® LIPIDOMIX® Mass Spec Standard (Catalog No. 
330707) consisting of 15:0-18:1(d7) PC, 15:0-18:1(d7) PE, 15:0-18:1(d7) PS, 15:0-18:1(d7) PG, 15:0-18:1(d7) PI, 15:0-18:1(d7) PA, 18:1(d7) LPC, 18:1(d7) LPE, 18:1(d7) Chol Ester, 18:1(d7) MG,15:0-18:1(d7) DG, 15:0-18:1(d7)-15:0 TG, 18:1(d9) SM, and cholesterol (d7) were from Avanti Polar Lipids (Alabaster, Alabama, USA). Standards including Cer(d18:1/16:0), Cer(d18:0/16:0), Cer(d18:1/18:0), Cer(d18:0/18:0), Cer(d18:1/24:0), Cer(d18:0/24:0), Cer(d18:1/24:1), Cer(18:0/24:1), Cer(18:2/24:0), sphinganine, sphingosine, sphiganine-1-P, sphingosine-1-P, CerP(d18:1/24:0), SM(d18:1/16:0), and SM(d18:1/24:0) were from Avanti Polar Lipids (Alabaster, Alabama, USA).

3.2 Blood and plasma collection.

Whole blood samples were collected from healthy controls (6 males, 4 females, average age 28.6) after overnight fasting into EDTA coated tubes (Catalog No. 367861, $4 \mathrm{~mL}$ tubes with $7.2 \mathrm{mg}$ EDTA). Each tube was gently inverted (without shaking or foaming contents) five times prior to transfer of $1 \mathrm{~mL}$ aliquots to separate LoBind tubes. All controls are enrolled in an ongoing natural history study at the Children's Hospital of Philadelphia (IRB \# 01-002609). Plasma was obtained from whole blood after centrifugation at $500 \times g$ for $20 \mathrm{~min}$ at $4{ }^{\circ} \mathrm{C}$.

\subsection{Sample preparation for sphingolipidomics}

\subsubsection{Butanol single phase extraction}

This method was used for the final extraction protocol for the whole blood and plasma. The method was modified according to previous papers[24,32]. Briefly, $50 \mu \mathrm{L}$ whole blood (or other volumes as indicated on graphs for the method development part) or plasma, mixed with $10 \mu \mathrm{L}$ internal standard solution (Cer/Sph Mixture I, was used as provided, and the concertation of each of the components is indicated in Table S1, as it was found from the Avanti website), were extracted with $1 \mathrm{~mL}$ butanol/methanol (1:1) $(v: v)$ with $10 \mathrm{mM}$ ammonium formate. After centrifugation at $500 \times g$ for $10 \mathrm{~min}$, the supernatant was collected and dried to completeness under a stream of nitrogen.

3.3.2 MTBE two phase extraction

$50 \mu \mathrm{L}$ whole blood or plasma, mixed with $10 \mu \mathrm{L}$ internal standard solution, were extracted with $0.75 \mathrm{~mL}$ methanol and $2.5 \mathrm{~mL} \mathrm{MTBE}$, then $0.625 \mathrm{~mL}$ water was added to induce two phase separation[33,34]. After centrifugation at $500 \times \mathrm{g}$ for $10 \mathrm{~min}$, the upper organic phase was transferred into a new tube and dried under a stream of nitrogen.

3.3.3 MTBE single phase extraction

This method was modified according to a published protocol (with chloroform being replaced by MTBE) to achieve better result for polar lipids[35]. Briefly, $50 \mu \mathrm{L}$ whole blood or plasma, mixed with $10 \mu \mathrm{L}$ internal standard solution, were extracted with 0.5 $\mathrm{mL}$ water, $1.25 \mathrm{~mL}$ methanol, and $1.25 \mathrm{~mL}$ MTBE. Different from the MTBE two phase method, this mixture would result in a final single phase for lipidomic analysis.

Each of the extraction steps was repeated, and the combined steps were dried to completeness under a stream of nitrogen. $100 \mu \mathrm{L}$ methanol/MTBE (1:3) (v:v) was used for the re-suspension for all of the extraction methods.

3.4 Alkaline hydrolysis

For the butanol single phase method, $100 \mu \mathrm{L} 0.1 \mathrm{M} \mathrm{KOH}$ was added to extraction solvent, and incubated at $37^{\circ} \mathrm{C}$ for $45 \mathrm{~min}$, then neutralized with $6 \mu \mathrm{L}$ glacial acetic acid (monitored with $\mathrm{pH}$ paper).

\subsection{Freeze/thaw cycle}

The freeze/thaw cycle was performed with fresh collected whole blood samples that were processed within 30 minutes of the blood draw. Fresh blood samples were aliquoted into $100 \mu \mathrm{L}$. Three technical replicates were used directly for extraction with butanol single phase method; the remaining aliquots were frozen at $-80^{\circ} \mathrm{C}$ for at least $24 \mathrm{~h}$. After $24 \mathrm{~h}$, the frozen blood samples were taken out of the freezer, and three technical replicates were extracted with butanol/methanol without thawing, while other three were thawed on ice $(\sim 2 h)$ and then processed with the same extraction method as for fresh blood. 


\subsection{Liquid chromatography-high resolution mass spectrometry (LC-HRMS)}

An Ultimate 3000 UPLC system coupled to a Q Exactive-HF mass spectrometer (both from Thermo Fisher Scientific, USA) were used for the sphingolipid analysis. Chromatographic separation of sphingolipids was achieved on an Accucore ${ }^{\mathrm{TM}}$ C18 column $(2.1 \times 150 \mathrm{~mm}, 2.6 \mu \mathrm{m})$ with solvent $\mathrm{A}$ as $0.1 \%$ formic acid in $10 \mathrm{mM}$ ammonium formate in acetonitrile/water $1 / 1(\mathrm{v} / \mathrm{v})$ and solvent $B$ as $0.02 \%$ formic acid in $2 \mathrm{mM}$ ammonium formate in acetonitrile/isopropanol/water 10/88/2 (v/v/v). The gradient was as follows: $0 \mathrm{~min}, 90 \% \mathrm{~A} ; 1 \mathrm{~min}, 90 \% \mathrm{~A} ; 4 \mathrm{~min}, 60 \% \mathrm{~A} ; 12 \mathrm{~min}, 25 \% \mathrm{~A} ; 21 \mathrm{~min}, 1 \% \mathrm{~A} ; 24 \mathrm{~min}$, $1 \% \mathrm{~A} ; 24.1 \mathrm{~min}, 90 \% \mathrm{~A} ; 28 \mathrm{~min}, 90 \% \mathrm{~A}$. The flow rate is $0.4 \mathrm{~mL} / \mathrm{min}$. Data acquisition was performed in full scan/ddMS2 mode @ 120,000 resolution in positive and negative mode, separately. The full scan settings were as follows: AGC target, 1e6; Maximum IT, 250 ms; scan range, 250 to $1800 \mathrm{~m} / \mathrm{z}$. Top $20 \mathrm{MS} / \mathrm{MS}$ spectral (dd-MS²) @ 15,000 were generated in the same cycle with AGC target 1e5, maximum IT $25 \mathrm{~ms}$, isolation window $1.0 \mathrm{~m} / \mathrm{z}$, and (N)CE/stepped nce 25, 30, 35v (positive mode).

Full scan/PRM or full scan/DIA were performed with scheduled inclusion list (Table S2). Full scan settings were as follows: $m / z$ 200-1500, resolution 120,000, Maximum IT 200 ms, AGC target 1e6. For PRM, spectra were acquired as follows: isolation window 1.0 $m / z$, resolution 15000, AGC 1e5, maximum IT 100ms. For DIA, spectra were acquired as follows: isolation window $1.0 \mathrm{~m} / \mathrm{z}$, resolution 15,000, AGC 1e5, maximum IT 100ms, loop count 20 .

\subsection{Lipidomics data analysis}

Peak detection, identification, alignment, and quantification were performed with LipidSearch 4.2 (Thermo Fisher Scientific, USA). Peak detection was achieved by searching their target database (HCD), and identification was performed using comprehensive ID algorithms (search type, Product) with precursor tolerance $=5 \mathrm{ppm}$, product tolerance $=10 \mathrm{ppm}, \mathrm{m}$-Score threshold $=2.0$. Identified peaks were filtered with $\mathrm{m}$-Score threshold (higher than 5.0) and ID quality filter (A and B and C). Adducts included $+\mathrm{H}$, $+\mathrm{NH} 4,+\mathrm{Na}$, and $+\mathrm{H}-\mathrm{H} 2 \mathrm{O}$ in positive mode and $-\mathrm{H},+\mathrm{HCOO},-2 \mathrm{H},-\mathrm{CH} 3$ in negative mode. Lipid results from each sample were then aligned within a RT window of $0.1 \mathrm{~min}$ and quantified by detecting their precursor ions from full MS and integrating XIC's.

\subsection{Data and Statistical analysis}

All the data were presented with mean \pm SD. Two-tailed unpaired t-test was used to compare the significant difference with $\mathrm{P}<0.05$. Graphpad Prism 9.0 was used to generate the illustrations and perform statistical analysis. Heatmap was conducted using R 3.6.3 with package pheatmap [36]. The approximate concentration for each compound was calculated by multiplying the area ratio of the compound to the relevant internal standard by the concentration of the internal standard added to each sample.

\section{Conclusions}

In this study, we developed a comprehensive and robust method for whole blood sphingolipidome profiling by combing single phase extraction, alkaline hydrolysis, appropriate internal standards correction, and ultra-high resolution $(R=120,000)$ mass spectrometry (DDA/PRM/DIA). Our results demonstrated that freeze/thaw process will not impact the stability of the sphingolipids in whole blood, implying that whole blood could be widely used for sphingolipid research. Comparison between whole blood and paired plasma from healthy controls indicated that whole blood had higher abundance sphingolipids than plasma, and whole blood showed much smaller individual variations in sphingolipid levels than plasma. All these results indicated that whole blood could be used as an alternative to plasma to guide sphingolipid biomarker discovery in clinical research, especially given the low volume of blood that could be used. Ceramides have been reported as promising biomarkers of cardiac events[37,38] and other pathologies as well[39], but their plasma concentrations varies depending on diet, time of day and other factors[40]. The RBC have a half-life of approximatively 120 days, so RBC sphingolipid 
measurements could reflect the average levels during the prior 4 months, which could be a better measure of the absolute sphingolipid levels than the fluctuating ones from plasma. Recent results suggested that sphingolipids overload in RBCs could occur during erythropoiesis[41]. Also as recently reported that differences at individual levels in plasma may favor the use of erythrocytes[42] our study investigated the best protocol to do that by using the minimum volume of whole blood and the relevant internal standards for normalization. We will move next to a case-control study of a particular disease to show the applicability of this new approach.

Supplementary Materials: The following are available online at www.mdpi.com/xxx/s1, Figure S1: The chromatogram and MS spectra of GM3(d34:1), Figure S2: Representative MS2 spectra of Cer(d18:1/24:0), Figure S3: Representative MS2 spectra of Hex1Cer(d18:1/24:0), Figure S4: Representative MS2 spectra of sphingosine-1-P in both positive (left) and negative (right) ion mode, Figure S5: Representative MS2 spectra of SM(d18:1/24:1) in both positive (left) and negative (right) ion mode, Figure S6: Representative MS2 spectra of GM3(d34:1) in both positive (left) and negative (right) ion mode, Figure S7: The MS2 product extracted chromatograms of Cer(d19:1/25:0) (lower channel, RT $16.63 \mathrm{~min}$ ) and Cer(d18:1/26:0) (middle channel, RT $16.83 \mathrm{~min}$ ) in data independent acquisition (DIA) mode, Figure S8: Representative MS2 spectra of CerP(d18:1/16:0) acquired from whole blood samples (left panel) or standard solution (right panel) in positive ion mode, Figure S9. Comparison of the raw peak intensity or normalized intensity (internal standard 12:0 ceramide) of ceramides, and their linear response vs different volume of blood, Figure S10: Comparison of the raw peak intensity or normalized intensity (internal standard 12:0 ceramide) of dihydroceramides, and their linear response vs different volume of blood, Figure S11: Comparison of the raw peak intensity or normalized intensity (internal standard C17 base SPHP) of SPHP, and their linear response vs different volume of blood, Figure S12: Comparison of the raw peak intensity or normalized intensity (internal standard C15 Hex1Cer-D7) of Hex1Cer, and their linear response vs different volume of blood. Figure S13: Comparison of the raw peak intensity or normalized intensity (internal standard C15 Hex1Cer-D7) of Hex2Cer, and their linear response vs different volume of blood. Figure S14: Comparison of the raw peak intensity or normalized intensity (internal standard C15 Hex1Cer-D7) of Hex3Cer, and their linear response vs different volume of blood. Figure S15: Comparison of the raw peak intensity or normalized intensity (internal standard 18:1 d7 SM) of $\mathrm{SM}$, and their linear response vs different volume of blood. Figure S16: Comparison of the raw peak intensity or normalized intensity (internal standard C18:0 GM3-d5) of GM3, and their linear response vs different volume of blood, Table S1: Ceramide/Sphingoid Internal Standard Mixture I, Table S2: Inclusion list for PRM experiments.

Author Contributions: Conceptualization, D.W. and C.M.; methodology, D.W. and C.M.; software, D.W.; validation, D.W. and C.M. and P.X.; formal analysis, D.W.; investigation, D.W. and P.X and C.M; resources, D.W. and C.M; data curation, D.W; writing-original draft preparation, D.W.; writing-review and editing, C.M.; visualization, C.M and D.W.; supervision, C.M.; project administration, C.M.; funding acquisition, C.M. All authors have read and agreed to the published version of the manuscript.

Funding: This research was funded by the NIH grants R21NS116315 and P30ES013508.

Institutional Review Board Statement: The study was conducted according to the guidelines of the Declaration of Helsinki, and approved by the Institutional Review Board of Children's Hospital of Philadelphia (protocol code IRB 01-002609 and date of approval 12-09-2020)

Informed Consent Statement: Informed consent was obtained from all subjects involved in the study.

Data Availability Statement: Data is contained within the article or supplementary material.

Acknowledgments: We thank Prof. Ian Blair for providing unlimited access for the use of LC-HRMS. We thank Lauren Hauser for the blood draw from all the heathy controls.

Conflicts of Interest: The authors declare no conflict of interest.

\section{References}

1. Nagana Gowda, G.; Raftery, D. Whole blood metabolomics by 1 H NMR spectroscopy provides a new opportunity to evaluate coenzymes and antioxidants. Analytical chemistry 2017, 89, 4620-4627. 
2. Stringer, K.A.; Younger, J.G.; McHugh, C.; Yeomans, L.; Finkel, M.A.; Puskarich, M.A.; Jones, A.E.; Trexel, J.; Karnovsky, A. Whole blood reveals more metabolic detail of the human metabolome than serum as measured by $1 \mathrm{H}-\mathrm{NMR}$ spectroscopy: implications for sepsis metabolomics. Shock (Augusta, Ga.) 2015, 44, 200.

3. Catalán, Ú.; Rodríguez, M.-Á.; Ras, M.-R.; Maciá, A.; Mallol, R.; Vinaixa, M.; Fernández-Castillejo, S.; Valls, R.-M.; Pedret, A.; Griffin, J.L. Biomarkers of food intake and metabolite differences between plasma and red blood cell matrices; a human metabolomic profile approach. Molecular BioSystems 2013, 9, 1411-1422.

4. Chaleckis, R.; Murakami, I.; Takada, J.; Kondoh, H.; Yanagida, M. Individual variability in human blood metabolites identifies age-related differences. Proceedings of the National Academy of Sciences 2016, 113, 4252-4259.

5. Chaleckis, R.; Ebe, M.; Pluskal, T.; Murakami, I.; Kondoh, H.; Yanagida, M. Unexpected similarities between the Schizosaccharomyces and human blood metabolomes, and novel human metabolites. Molecular BioSystems 2014, 10, 2538-2551.

6. Speziale, R.; Montesano, C.; De Leonibus, M.L.; Bonelli, F.; Fezzardi, P.; Beconi, M.G.; Monteagudo, E.; Elbaum, D.; Orsatti, L. Determination of acetyl coenzyme A in human whole blood by ultra-performance liquid chromatography-mass spectrometry. Journal of Chromatography B 2018, 1083, 57-62.

7. Song, J.; Liu, X.; Wu, J.; Meehan, M.J.; Blevitt, J.M.; Dorrestein, P.C.; Milla, M.E. A highly efficient, high-throughput lipidomics platform for the quantitative detection of eicosanoids in human whole blood. Analytical biochemistry 2013, 433, 181-188.

8. Marasca, C.; Arana, M.E.B.; Protti, M.; Cavalli, A.; Mercolini, L.; Armirotti, A. Volumetric Absorptive Microsampling of Blood for Untargeted Lipidomics. Molecules 2021, 26, doi:10.3390/molecules26020262.

9. Lu, W.H.; Chiu, H.H.; Kuo, H.C.; Chen, G.Y.; Chepyala, D.; Kuo, C.H. Using matrix-induced ion suppression combined with LC-MS/MS for quantification of trimethylamine-N-oxide, choline, carnitine and acetylcarnitine in dried blood spot samples. Anal Chim Acta 2021, 1149, 338214, doi:10.1016/j.aca.2021.338214.

10. Gao, F.; McDaniel, J.; Chen, E.Y.; Rockwell, H.E.; Drolet, J.; Vishnudas, V.K.; Tolstikov, V.; Sarangarajan, R.; Narain, N.R.; Kiebish, M.A. Dynamic and temporal assessment of human dried blood spot MS/MS(ALL) shotgun lipidomics analysis. Nutr Metab (Lond) 2017, 14, 28, doi:10.1186/s12986-017-0182-6.

11. Aristizabal Henao, J.J.; Metherel, A.H.; Smith, R.W.; Stark, K.D. Tailored Extraction Procedure Is Required To Ensure Recovery of the Main Lipid Classes in Whole Blood When Profiling the Lipidome of Dried Blood Spots. Anal Chem 2016, 88, 9391-9396, doi:10.1021/acs.analchem.6b03030.

12. Kondoh, H.; Kameda, M.; Yanagida, M. Whole Blood Metabolomics in Aging Research. Int J Mol Sci 2020, 22, doi:10.3390/ijms22010175.

13. Lahiri, S.; Futerman, A. The metabolism and function of sphingolipids and glycosphingolipids. Cellular and Molecular Life Sciences 2007, 64, 2270-2284.

14. Hannun, Y.A.; Obeid, L.M. Sphingolipids and their metabolism in physiology and disease. Nature reviews Molecular cell biology 2018, 19, 175.

15. Couttas, T.A.; Kain, N.; Tran, C.; Chatterton, Z.; Kwok, J.B.; Don, A.S. Age-dependent changes to sphingolipid balance in the human hippocampus are gender-specific and may sensitize to neurodegeneration. Journal of Alzheimer's Disease 2018, 63, 503-514.

16. Ellis, B.; Hye, A.; Snowden, S.G. Metabolic modifications in human biofluids suggest the involvement of sphingolipid, antioxidant, and glutamate metabolism in Alzheimer's disease pathogenesis. Journal of Alzheimer's Disease 2015, 46, 313-327.

17. Futerman, A.H.; Riezman, H. The ins and outs of sphingolipid synthesis. Trends in cell biology 2005, 15, 312-318.

18. Futerman, A.H. Intracellular trafficking of sphingolipids: relationship to biosynthesis. Biochimica Et Biophysica Acta (BBA)-Biomembranes 2006, 1758, 1885-1892. 
19. Huwilera, A. Physiology and pathophysiology of sphingolipid metabolism and signaling. Biochim. Biophys. Acta 2000, 1485, 63-99.

20. Sullards, M.C.; Liu, Y.; Chen, Y.; Merrill Jr, A.H. Analysis of mammalian sphingolipids by liquid chromatography tandem mass spectrometry (LC-MS/MS) and tissue imaging mass spectrometry (TIMS). Biochimica et Biophysica Acta (BBA)-Molecular and Cell Biology of Lipids 2011, 1811, 838-853.

21. Sullards, M.C.; Allegood, J.C.; Kelly, S.; Wang, E.; Haynes, C.A.; Park, H.; Chen, Y.; Merrill Jr, A.H. Structure - Specific, quantitative methods for analysis of sphingolipids by liquid Chromatography-Tandem mass spectrometry:"Inside - Out” sphingolipidomics. Methods in enzymology 2007, 432, 83-115.

22. Peng, B.; Weintraub, S.T.; Coman, C.; Ponnaiyan, S.; Sharma, R.; Tews, B.r.; Winter, D.; Ahrends, R. A comprehensive high-resolution targeted workflow for the deep profiling of sphingolipids. Analytical chemistry 2017, 89, 12480-12487.

23. Quehenberger, O.; Armando, A.M.; Brown, A.H.; Milne, S.B.; Myers, D.S.; Merrill, A.H.; Bandyopadhyay, S.; Jones, K.N.; Kelly, S.; Shaner, R.L. Lipidomics reveals a remarkable diversity of lipids in human plasma. Journal of lipid research 2010, 51, 3299-3305.

24. Huynh, K.; Barlow, C.K.; Jayawardana, K.S.; Weir, J.M.; Mellett, N.A.; Cinel, M.; Magliano, D.J.; Shaw, J.E.; Drew, B.G.; Meikle, P.J. High-throughput plasma lipidomics: detailed mapping of the associations with cardiometabolic risk factors. Cell chemical biology 2019, 26, 71-84. e74.

25. Zhou, W.; Yang, S.; Wang, P.G. Matrix effects and application of matrix effect factor. Future Science: 2017.

26. Hammad, S.M.; Pierce, J.S.; Soodavar, F.; Smith, K.J.; Al Gadban, M.M.; Rembiesa, B.; Klein, R.L.; Hannun, Y.A.; Bielawski, J.; Bielawska, A. Blood sphingolipidomics in healthy humans: impact of sample collection methodology. Journal of lipid research 2010, 51, 3074-3087.

Brunkhorst, R.; Pfeilschifter, W.; Patyna, S.; Büttner, S.; Eckes, T.; Trautmann, S.; Thomas, D.; Pfeilschifter, J.; Koch, A. Preanalytical biases in the measurement of human blood Sphingolipids. International journal of molecular sciences 2018, 19, 1390.

28. Capiau, S.; Wilk, L.S.; De Kesel, P.M.M.; Aalders, M.C.G.; Stove, C.P. Correction for the Hematocrit Bias in Dried Blood Spot Analysis Using a Nondestructive, Single-Wavelength Reflectance-Based Hematocrit Prediction Method. Anal Chem 2018, 90, 1795-1804, doi:10.1021/acs.analchem.7b03784.

29. Lamers, Y.; Prinz-Langenohl, R.; Brämswig, S.; Pietrzik, K. Red blood cell folate concentrations increase more after supplementation with [6S]-5-methyltetrahydrofolate than with folic acid in women of childbearing age. Am J Clin Nutr 2006, 84, 156-161, doi:10.1093/ajcn/84.1.156.

30. Sato, K.; Katsumata, Y.; Aoki, M.; Suzuki, O.; Kido, A.; Oya, M.; Yada, S. A new reagent for the rapid determination of total hemoglobin as hemiglobincyanide in blood containing carboxyhemoglobin. Biochem Med 1983, 30, 78-88, doi:10.1016/0006-2944(83)90010-8.

31. Huang, Y.; Khartulyari, S.; Morales, M.E.; Stanislawska-Sachadyn, A.; Von Feldt, J.M.; Whitehead, A.S.; Blair, I.A. Quantification of key red blood cell folates from subjects with defined MTHFR 677C>T genotypes using stable isotope dilution liquid chromatography/mass spectrometry. Rapid Commun Mass Spectrom 2008, 22, 2403-2412, doi:10.1002/rcm.3624.

32. Alshehry, Z.H.; Barlow, C.K.; Weir, J.M.; Zhou, Y.; McConville, M.J.; Meikle, P.J. An efficient single phase method for the extraction of plasma lipids. Metabolites 2015, 5, 389-403.

33. Ulmer, C.Z.; Jones, C.M.; Yost, R.A.; Garrett, T.J.; Bowden, J.A. Optimization of Folch, Bligh-Dyer, and Matyash sample-to-extraction solvent ratios for human plasma-based lipidomics studies. Analytica chimica acta 2018, 1037, $351-357$.

34. Wong, M.; Braidy, N.; Pickford, R.; Sachdev, P.; Poljak, A. Comparison of single phase and biphasic extraction protocols for lipidomic studies using human plasma. Frontiers in neurology 2019, 10, 879. 
35. Lydic, T.A.; Busik, J.V.; Reid, G.E. A monophasic extraction strategy for the simultaneous lipidome analysis of polar and nonpolar retina lipids. Journal of lipid research 2014, 55, 1797-1809.

36. Kolde, R. Pheatmap: pretty heatmaps. R package version 2012, 1.

37. Kurz, J.; Parnham, M.J.; Geisslinger, G.; Schiffmann, S. Ceramides as Novel Disease Biomarkers. Trends Mol Med 2019, 25, 20-32, doi:10.1016/j.molmed.2018.10.009.

38. Tippetts, T.S.; Holland, W.L.; Summers, S.A. The ceramide ratio: a predictor of cardiometabolic risk. J Lipid Res 2018, 59, 1549-1550, doi:10.1194/jlr.C088377.

39. McGrath, E.R.; Himali, J.J.; Xanthakis, V.; Duncan, M.S.; Schaffer, J.E.; Ory, D.S.; Peterson, L.R.; DeCarli, C.; Pase, M.P.; Satizabal, C.L., et al. Circulating ceramide ratios and risk of vascular brain aging and dementia. Ann Clin Transl Neurol 2020, 7, 160-168, doi:10.1002/acn3.50973.

40. Brunkhorst, R.; Pfeilschifter, W.; Patyna, S.; Büttner, S.; Eckes, T.; Trautmann, S.; Thomas, D.; Pfeilschifter, J.; Koch, A. Preanalytical Biases in the Measurement of Human Blood Sphingolipids. Int J Mol Sci 2018, 19, doi:10.3390/ijms19051390.

41. Dupuis, L.; Chipeaux, C.; Bourdelier, E.; Martino, S.; Reihani, N.; Belmatoug, N.; Billette de Villemeur, T.; Hivert, B.; Moussa, F.; Le Van Kim, C., et al. Effects of sphingolipids overload on red blood cell properties in Gaucher disease. J Cell Mol Med 2020, 24, 9726-9736, doi:10.1111/jcmm.15534.

42. Loef, M.; von Hegedus, J.H.; Ghorasaini, M.; Kroon, F.P.B.; Giera, M.; Ioan-Facsinay, A.; Kloppenburg, M. Reproducibility of Targeted Lipidome Analyses (Lipidyzer) in Plasma and Erythrocytes over a 6-Week Period. Metabolites 2020, 11, doi:10.3390/metabo11010026. 